\title{
Methods of Sensitivity Analyses and their Role in Engineering
}

\author{
NOVÁK Drahomír*, LEHKÝ David \\ Faculty of Civil Engineering, Institute of Structural Mechanics \\ Brno University of Technology \\ 60200 Brno, Czech Republic \\ anovak.d@fce.vutbr.cz
}

\author{
CAO Maosen, PAN Lixia \\ Department of Engineering Mechanics, College of Mechanics \\ and Materials, Hohai University, \\ Nanjing 210098, People's Republic of China
}

\begin{abstract}
The paper describes selected efficient methods for sensitivity analysis of engineering problems: The non-parametric rank-order statistical correlation between the basic random variables and the structural response variable, neural network ensemble-based sensitivity analysis and sensitivity analysis in terms of coefficient of variation. All three methods were utilized and compared for practical engineering example.
\end{abstract}

Keywords-sensitivity analysis, artificial neural networks, reliability analysis

\section{INTRODUCTION}

Sensitivity analysis (SA) is a crucial step in the computational modelling and assessment. SA is important step in every simulation and assessment, therefore it has received much attention in the literature in the past decade. Through sensitivity analysis we gain essential insights on computational model behavior, on its structure and on its response to changes in the model inputs. Sensitivity analysis of a model aims at quantifying the relative importance of each input parameter. Such SA is important in order to reduce space of random variables for stochastic calculation, building of response surface, training of neural networks, etc. Several interrogations are possible and several SA methods have been developed, giving rise to a vast and growing literature. Overview of available methods is given in review papers, e.g. [1-4]. Versatile sensitivity indices for nonlinear non-monotonic problems are known to be based on Sobol' decomposition of functions [5, 6]. Sudret [7] introduced generalized polynomial chaos expansions in order to build surrogate models that allow to compute the Sobol' indices analytically. Artificial neural networks (ANNs) are generally used in the civil engineering field as powerful, flexible, versatile techniques for the prediction, classification and regression of engineering variables. Apart from these prevailing applications, the use of ANNs to perform parameter SA for engineering systems is still lacking [8], although the huge potential of ANNs has become evident in this new area of research. Representative studies of the use of ANNs to analyze the sensitivity of parameters of an engineering system are e.g. [9-12]. The paper shows alternative SA techniques and theirs utilization for practical engineering example.

The authors give thanks for the financial support of the Czech Science Agency project No. 17-02862S ("PROMOSS") and the Belt and Road project.

\section{SIMULATION-BASED SA: NON-PARAMETRIC RANK-ORDER AND IN THE FORM OF COV}

The methodology for stochastic modelling Latin Hypercube Sampling (LHS) [13, 14] is well known nowadays. LHS belongs to the category of advanced stratified Monte Carlo type sampling which results in very good estimation of statistical moments of response using a small-sample simulation. More accurately, LHS is considered to be one of the variance reduction techniques, because it yields lower variance of estimates of statistical moments compared to crude Monte Carlo sampling at the same sample size, see e.g. [15]. This is the reason the technique became very attractive when dealing with computationally intensive problems like e.g. complex finite element method (FEM) simulations - and this is the case of complex FEM modelling, especially in 3D. With respect to the small-sample simulation technique described above the straightforward and the simplest approach uses the non-parametric rank-order statistical correlation between the basic random variables and the structural response variable [16, 17]. The sensitivity analysis is obtained as an additional result of LHS, and no additional computational effort is necessary. The relative effect of each basic variable on the structural response can be measured using the partial correlation coefficient between each basic input variable and the response variable. Because the model for the structural response is generally non-linear, a non-parametric rank-order correlation is used by means of the Spearman correlation coefficient or Kendall tau. This type of SA represent relative measures of variables importance. A very practical type of SA is sensitivity in the form of coefficient of variation (COV). When using Monte Carlo type simulation, simulated set of structural response variable is statistically evaluated and the coefficient of variation $C O V$ can be found. Let us designate the partial coefficient of variation $C O V_{R i}$ for the case in which the variable $X_{i}$ is the only one treated random in the simulation process. The other basic variables are kept at their mean values. The partial factor for the influence of variable $X_{i}$ may be defined as

$$
\alpha_{i}=\frac{C O V_{R i}}{C O V_{X i}}
$$


where, $C O V_{X i}$ is the coefficient of variation of basic random variable $X_{i}$. These types of SA are implemented in reliability software FReET $[17,18]$, widely used in engineering.

\section{ANN-BASED SA: NEURAL NETWORK ENSEMBLE-BASED PARAMETER SENSITIVITY ANALYSIS}

The use of artificial neural networks for parameter sensitivity analysis in civil engineering systems is an emerging research focus of increased interest. Existing methods are generally based on a single neural network, but are inadequate as a basis for parameter sensitivity analysis because of the instability of a single neural network. To address this deficiency, development of a neural network ensemble-based parameter sensitivity analysis paradigm is proposed. This paradigm features use of a set of preselected superior neural networks to make decisions about parameter sensitivity by averaging the sensitivity analysis results of individual neural networks. The proposed method provides a common paradigm to analyze the sensitivity of influential parameters, shedding light on the underlying mechanisms of civil engineering systems. The NNE-based parameter sensitivity analysis paradigm represents an alternative way to perform sensitivity analysis [19]. This paradigm involves four basic steps: (i) empirically choose a sophisticated type of neural network as the seed; (ii) the seed produces a family of $k$ candidate neural networks with dissimilar network structures; (iii) by removing the inferior neural networks, $n(n \leq k)$ superior neural networks with better performance are selected to form a NNE model; (iv) a selected sensitivity analysis algorithm is implemented on the NNE model to perform parameter sensitivity analysis, leading to a ranking of all the factors pertaining to the engineering system of interest. Fig. 1 shows a schematic of NNE-based parameter sensitivity analysis, where the components of seed, candidates, NNE model and ranking are clearly identified. The application of the procedure can be found e.g. in $[12,20,21]$.

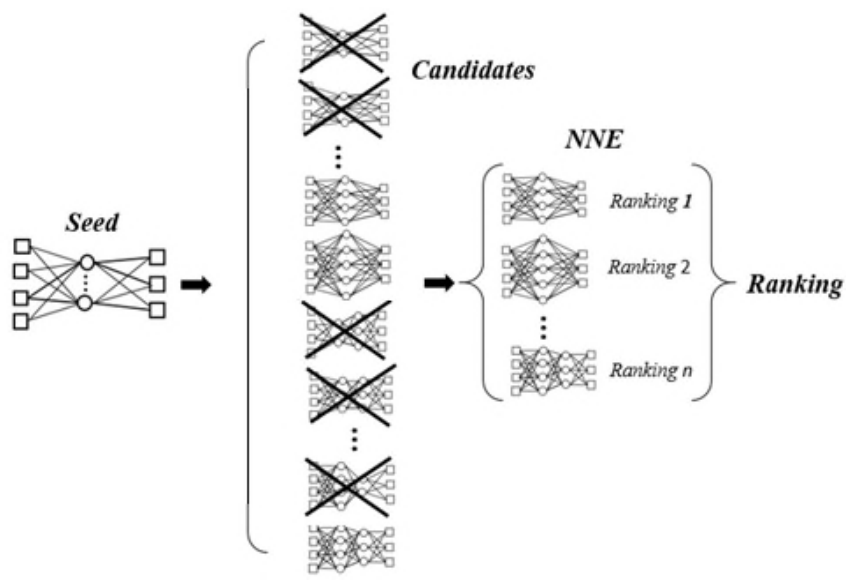

Fig. 1. NNE-based parameter sensitivity.

\section{ILLUSTRATIVE EXAMPLE}

The methods have been applied and compared recently for a single-span post-tensioned composite bridge located in South Moravia region in the Czech Republic, FEM computational model was nonlinear and therefore computational effort high, for details see [21]. For limit state of crack initiation six dominant parameters were identified, see Fig. 2. Parameters with high-level importance are prestress force $P_{1}$ (Spearman sensitivity is 0.61 , normalized is 1 for Spearman and COV methods, 0.95 for NNE method), tensile strength of joints $f_{\mathrm{t}}$ (Spearman sensitivity is 0.59 , normalized values are 0.98 for Spearman, 1 for NNE, and 0.83 for COV) and fracture energy of joints $G_{\mathrm{f}}$ (Spearman sensitivity is 0.51 , normalized are 0.84 for Spearman, 0.11 for NNE and 0.003 for COV method). Parameter with middle-level importance is prestress force $P_{2}$ (Spearman sensitivity is 0.378 , normalized values are 0.6 for Spearman and NNE, and 0.4 for COV). Parameters with low-level importance are compressive strength of joints $f_{\mathrm{c}}$ and secondary dead load $g_{1}$. Other parameters have sensitivities lower then 0.1 ( 0.1 normalized) and can be declared as almost zero-level importance.

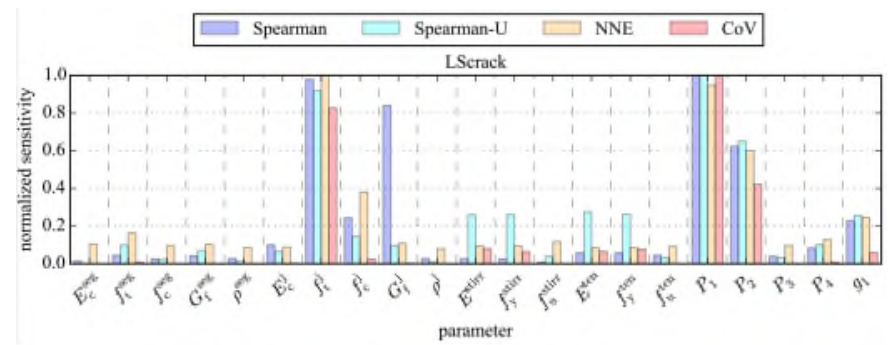

Fig. 2. Normalized sensitivity measures (Spearman, COV, NNE).

\section{SUMMARY}

The paper briefly summarizes methods for sensitivity analysis of engineering problems. The attention is focused on simulation-based sensitivity and ANN-based sensitivity measures. They play a significant role in engineering, where distinguish dominant and non-dominant variables is important for: Design; Optimization; Reliability assessment; Quality control; Building meta-models, etc.

\section{REFERENCES}

[1] D. Novák, B. Teplý, N. Shiraishi, Sensitivity analysis of structures: A review. In Proc. Of Int. Conference CIVIL COMP'93: Edinburgh, Scotland, 1993, pp. 201-207.

[2] J.P.C Kleijnen, "Sensitivity Analysis of Simulation Models: an Overview”. Proc. Soc. Behav. Sci. vol. 2, pp. 7585-7586, 2010.

[3] E. Borgonovo, E. Plischke, "Sensitivity analysis: A review of recent advances”. European J. Operat. Res. vol. 248, pp. 869-887, 2016.

[4] J. Antucheviciene, Z. Kala, M. Marzouk, E.R. Vaidogas, "Solving civil engineering problems by means of fuzzy and stochastic MCDM methods: Current state and future research. Math. Probl. Eng”. 362579, pp. 1-16, 2015.

[5] I.M. Sobol, Sensitivity analysis for non-linear mathematical models. Math. Modelling and Computational Experiment, 1, 1993, 407-414. Transl. from Russian: I. M. Sobol'. 1990. Sensitivity estimates for nonlinear mathematical models, Matematicheskoe Modelirovanie 2: 112 118.

[6] I. M Sobol, "Global sensitivity indices for nonlinear mathematical models and their Monte Carlo estimates". Math. Comput. Simul. vol. 55, pp. 271280, 2001.

[7] B. Sudret, "Global sensitivity analysis using polynomial chaos expansions". Reliabil. Eng. Syst. Saf. vol. 93, no. 964-979, 2008.

[8] J.D. Olden, D.A. Jackson, "Illuminating the "black box": a randomization approach for understanding variable contributions in artificial neural networks". Ecol. Model. vol. 154, no. 1, pp. 135-150, 2002.

[9] S.K. Das, R.K. Biswal, N. Sivakugan, B. Das, "Classification of slopes and prediction of factor of safety using differential evolution neural networks". Environ. Earth Sci. vol. 64, no. 1, pp. 201-210, 2011. 
[10] M. Hadzima-Nyarko, E.K. Nyarko, D. Morić, "A neural network based modelling and sensitivity analysis of damage ratio coefficient". Expert Syst. Appl. vol. 38, no. 10, pp. 13405-13413, 2011.

[11] A. Jain, S.K. Jha, S. Misra, "Modeling and analysis of concrete slump using artificial neural networks". J. Mater. Civil Eng. vol. 20, no. 9, 628-633, 2008.

[12] M.S. Cao, L.X. Pan, Y.F, Gao, D. Novák, Z.C. Ding, D. Lehký, X.L. Li, "Neural network ensemble-based parameter sensitivity analysis in civil engineering systems". Neural Comp. App. 26 (2015) 8.

[13] M.D. McKay, W.J. Conover, R.J. Beckman, "A comparison of three methods for selecting values of input variables in the analysis of output from a computer code". Technometr. vol. 21, pp. 239-245, 1979.

[14] M. Stein, "Large sample properties of simulations using Latin hypercube sampling". Technometr. vol. 29, no. 2, pp. 143-151, 1987.

[15] J.R. Koehler, A.B. Owen, Computer experiments, Ghosh S, Rao CR. (Eds.) Handbook of Statistics, 1996, 261-308, Elsevier Science, New York, vol. 13, pp. 261-308, 1996.

[16] R.C. Iman, W.J. Conover, "Small Sample Sensitivity Analysis Techniques for Computer Models, with an Application to Risk Assessment". Commun. Stat. Theor. Meth. vol. A9, pp. 1749-1842, 1980.

[17] D. Novák, M. Vořechovský, B. Teplý, "FReET: Software for the statistical and reliability analysis of engineering problems and FReET-D: Degradation module”. Adv. Eng. Softw. vol. 72, pp. 179-192, 2014.

[18] D. Novák, M. Vořechovský, R. Rusina, FREET version 1.6 - program documentation, User's and Theory Guides, Brno/Červenka Consulting, Prague; 2017, http://www.freet.cz.

[19] M.S. Cao, P.Z. Qiao, "Neural network committee-based parameter sensitivity analysis paradigm for geotechnical engineering problems". Neural Comp. Appl. vol. 17, no. (5-6), 509-519, 2008.

[20] D. Novák, D. Lehký, L. Pan, M. Cao, Sensitivity analysis strategies for artificial neural networks modelling of engineering problems. In: Proceedings of the International Conference on Civil, Urban and Environmental Engineering (CUEE2014), Beijing, China, 2014, pp. 161-169.

[21] D. Lehký, D. Novák, M. Šomodíková, L. Pan, M. Cao, A Comparison of Sensitivity Analyses of Prestressed Composite Bridge. In: Proceedings of the International Conference on Structural Safety and Reliability Icossar 2017, Vienna, Austria, 2017. 